\title{
SUSTITUTIVOS ÓSEOS
}

\section{Ó. Fernández Hernández}

Servicio de Cirugía Ortopédica y Traumatología.

Complejo Asistencial Universitario de León

\section{Introducción}

El tratamiento de los defectos óseos es un continuo reto en la traumatología ${ }^{(1)}$. Los sustitutivos óseos son materiales naturales, inorgánicos o sintéticos que pueden ser introducidos para el tratamiento de los defectos óseos como alternativa al hueso autólogo u homólogo ${ }^{(2)}$. El sustitutivo óseo ideal no debe producir respuesta inflamatoria, debe ser biocompatible, fácilmente moldeable dentro del defecto óseo, osteoconductivo, osteoinductivo y reabsorbible. Además, debe ser no termoconductor, esterilizable y fácilmente asequible a un coste razonable ${ }^{(3-5)}$. Los sustitutivos óseos se pueden clasificar en injertos óseos (autoinjertos, homoinjertos, xenoinjertos), cerámicas (hidroxiapatita -HA-, sulfato cálcico...) y factores de crecimiento (proteínas morfogenéticas óseas -BMP, bone morphogenetic proteins-, plasma rico en plaquetas -PRP-, matriz ósea desmineralizada -DBM, demineralised bone matrix-...) (2,5) (Figura 1). Están disponibles en múltiples tamaños y formas, como chips, pasta, polvo, gel...(5) (Figura 2).

La osteointegración de un injerto o de otro sustitutivo óseo depende de sus propiedades osteogénicas, osteoinductoras y osteoconductoras que, todas presentes en los autoinjertos, hacen de estos los injertos óseos ideales ${ }^{(3-6)}$ (Figura 3):

- Osteogénesis: capacidad para generar hueso desde células óseas. El injerto óseo osteogénico más utilizado es el autoinjerto, que tiene elementos celulares y factores de crecimiento.

- Osteoinducción: estimulación y activación de células mesenquimales pluripotenciales del tejido circundante, que se diferencian en osteoblastos. Diferentes factores de crecimiento actúan en el proceso osteoinductivo.

- Osteoconducción: capacidad de crear una nueva red vascular y un nuevo sistema haversiano en el andamiaje.

FS $\odot 2017$ SEMCPT. Publicado por Imaidea Interactiva en FONDOSCIENCE ${ }^{\circledR}$ (www.fondoscience.com). 


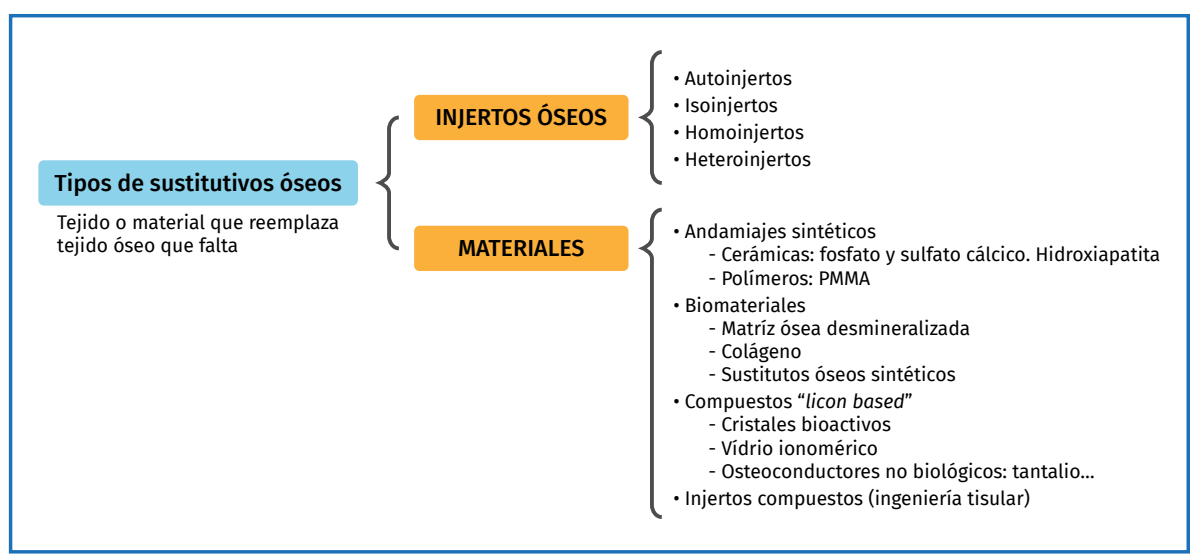

Figura 1. Tipos de sustitutivos óseos. PMMA: polimetilmetacrilato. génicas, sin riesgos inmunogénicos o de infección. Las principales desventajas son la morbilidad de la zona donante (hematomas, dolor residual, fracturas...) y su limitada disponibilidad. Aunque el autoinjerto de cresta iliaca es el gold standard dentro de los autoinjertos óseos, hay una emergente evidencia que apoya el injerto obtenido mediante sistema RIA (reamer-irrigator-aspirator): puede proporcionar grandes volúmenes de injerto de manera eficiente, con una morbilidad menor en el sitio donante ${ }^{(9,10)}$.

\section{Homoinjertos}

Resultan de la obtención de hueso de un individuo de la misma especie, bien vivo (cabeza femoral en prótesis totales de cadera) o de cadáver (donación ósea), y deben ser procesados en un banco de tejidos. Los homoinjertos frescos son osteogénicos, osteoinductivos y osteoconductivos, pero tienen riesgo de transmisión de enfermedades e inmunogenicidad asociada. El homoinjerto congelado es un tejido osteoconductivo, pero con leve osteoinducción debido a la pérdida de factores de crecimiento según la técnica de conservación y con pérdida de sus propiedades mecánicas originales. Los homoinjertos liofilizados pierden su celularidad en el procesado, por lo que no son osteogénicos. Los homoinjertos liofilizados son el tipo más común de homoinjerto utilizado actualmente en la cirugía de pie y tobillo(5). Las limitaciones de los homoinjertos son su coste, el procesado laborioso, la resistencia mecánica, su limitada osteoinducción y el riesgo potencial de transmisión de enfermedades del donante.

\section{Xenoinjertos}

Los xenoinjertos proporcionan soporte estructural y osteoconductor, pero no son osteogénicos ni osteoinductores. Resultan de la obtención de tejido óseo de una especie diferente a la humana, tal 


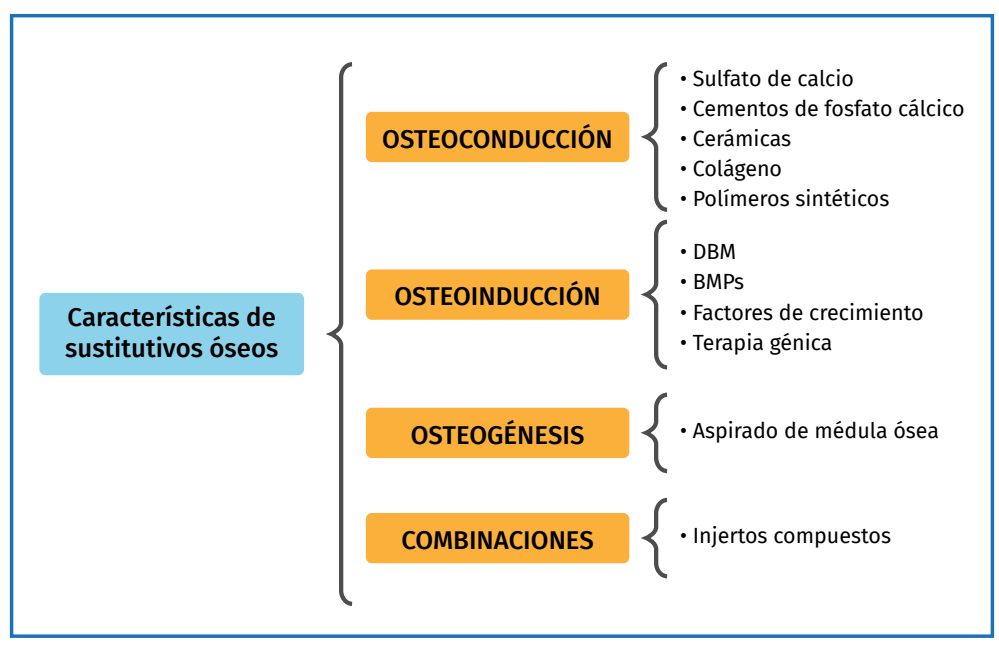

Figura 3. Características de los sustitutivos óseos. BMP: proteínas morfogenéticas óseas; DBM: matriz ósea desmineralizada. sidad diferente y resistencia mecánica limitada(4), no suficiente para su aplicación en zonas de carga ${ }^{(7,11)}$.

Los sustitutivos coralinos pueden ser naturales o artificiales. Algunas especies de coral marino producen una estructura hecha de fosfato cálcico similar al hueso esponjoso humano. La HA coralina se obtiene de la forma natural ${ }^{(3,5)}$, mediante la conversión del carbonato cálcico de la estructura del coral en $\mathrm{HA}^{(12)}$, y está disponible en bloques o gránulos. Su uso está limitado por sus características mecánicas y su falta de reabsorción, y sus ventajas son la ausencia de transmisión de enfermedades, la compatibilidad inmunológica y la osteoconducción ${ }^{(12)}$. La HA nanocristalina se ha como el hueso porcino o bovino. Los xenoinjertos coralinos son principalmente carbonatos cálcicos, mientras que el hueso humano está hecho de HA con fosfatos y carbonatos cálcicos.

\section{Hidroxiapatita y fosfato tricálcico}

Son compuestos minerales basados en el fosfato de calcio. Pueden ser preparados como cerámicas. Forman osteoide al ponerse en contacto con el hueso, que se mineraliza y se reabsorbe, sustituyéndose por hueso. Se pueden fabricar en una gran variedad de formas, incluyendo gránulos, bloques porosos o como recubrimiento de implantes.

El fosfato tricálcico (TCP, tricalcium phosphate) tiene altas propiedades de osteoconducción y se reabsorbe fácilmente(6). Los poros en los materiales de fosfato cálcico son necesarios para permitir la migración y proliferación de vasos, osteoblastos y células mesenquimales, siendo su mínimo tamaño recomendado de $100 \mu \mathrm{m}$. La excelente biocompatibilidad y propiedades osteoconductivas de las cerámicas de fosfato cálcico las han convertido en la tercera mejor opción en la cirugía de reconstrucción ósea, por detrás de los autoinjertos y los homoinjertos ${ }^{(11)}$. La HA se mantiene estable durante largo tiempo y tiene buenas propiedades mecánicas. No poseen capacidad osteogénica ni osteoinductiva, pero sí osteoconductiva, con suministro ilimitado y disponibilidad en todas las formas y tamaños, incluso por encargo, poro- utilizado con buenos resultados como relleno de defectos óseos en fracturas de calcáneo tratadas mediante reducción abierta y fijación interna con $\operatorname{placa}^{(13)}$.

Las biocerámicas por sí solas carecen de propiedades osteogénicas que les permitan reparar grandes defectos óseos, por lo que actualmente la investigación se centra en impartirles propiedades regenerativas óseas similares a los autoinjertos $^{(11)}$. Los materiales de fosfato cálcico combinados con BMP, células osteoprogenitoras y proteínas o péptidos bioactivos mejoran la formación ósea porque producen una osteoinductividad diseñada ${ }^{(7)}$.

\section{Cementos de fosfato cálcico (CFC)}

Los CFC pueden ser inyectados, fraguan en unos 20 minutos formando HA nanocristalina, no desprenden calor y pueden servir de transportador para factores de crecimiento, antibióticos y $\operatorname{BMP}^{(2,14)}$. Existen diferentes tipos, como las apatitas (HA) y las brushitas (dihidrato-fosfato dicálcico)(15). Ambos se producen mezclando ortofosfatos cálcicos con una solución acuosa. Uno de sus inconvenientes es su fragilidad, por lo que no pueden ser aplicados para soportar carga. Sin embargo, pueden ser fácilmente introducidos en cavidades debido a sus consistencia de gel. Otro posible problema es que podrian reabsorberse antes de producirse la neoformación ósea ${ }^{(1)}$, siendo las brushitas las 
de más rápida absorción ${ }^{(15)}$. Las investigaciones en relación con el CFC se han centrado en mejorar sus propiedades mecánicas( ${ }^{(2,15)}$, de inyectabilidad $^{(15)}$ y de reabsorción/incorporación ${ }^{(10)}$. En un reciente estudio de sus características, solo el Eurobone ${ }^{\circledR}$ tiene poros mayores de $100 \mu \mathrm{m}$, que es lo que se ha establecido como necesario para una correcta osteoconducción ${ }^{(1)}$.

\section{Sulfato de calcio (SC)}

Entre sus propiedades destacan su fácil obtención, capacidad de osteoconducción, rápida absorción y fácil modelado. Sin embargo, diferencias en la morfología y estructura de sus cristales, en la porosidad, en las propiedades mecánicas y en la constitución química del SC pueden alterar significativamente sus propiedades biológicas ${ }^{(2,5,16)}$. Además, se han descubierto muchos efectos adversos y ausencia de efectos, en parte debidos a su rápida resorción. Por ello, el SC se ha propuesto como un andamiaje para la DBM. La incorporación de carbonato cálcico al SC puede disminuir la tasa de degradación e inducir una más rápida formación ósea, según estudios en ratas ${ }^{(17)}$. Su utilización como sustitutivo óseo es muy limitada, siendo su mejor uso el transporte de antibióticos adyuvantes $^{(9)}$.

\section{Compuestos de colágeno e hidroxiapatita (Col-HA)}

El colágeno y la HA combinados aceleran la osteogénesis ${ }^{(2)}$. Las propiedades dúctiles del colágeno aumentan la mala resistencia a la fractura de las HA. El colágeno contribuye al depósito de minerales, al crecimiento vascular y a la unión a factores de crecimiento, proporcionando un entorno favorable para la regeneración ósea ${ }^{(3)}$. La adición de fosfato de calcio al Col-HA le proporciona mayor estabilidad y resistencia.

\section{Compuestos licon-based}

\section{Cristales bioactivos}

Los cristales bioactivos son materiales duros y sólidos, hechos de calcio, fósforo y dióxido de silicio (silicatos) $)^{(14)}$, con propiedades osteoconductivas. Los bloques de cristal bioactivo pueden perforarse y conformarse; sin embargo, se pueden romper en el proceso, por lo que son difíciles de usar en el esqueleto. Las cerámicas bioactivas, una nueva variación, son más fuertes que el cristal bioactivo, con propiedades mecánicas mejoradas. La incorporación de fibras de acero inoxidable y partículas cerámicas (zirconia) aumentan la resistencia a la flexión y la dureza ${ }^{(3)}$.

\section{Ionómeros de cristal}

El cemento ionomérico es una pasta de calcio, aluminio y fluorosilicato, que endurece en $5 \mathrm{mi}$ nutos y después de 24 horas tiene una resistencia a la compresión y un módulo de elasticidad comparable al hueso cortical. Su estructura porosa ayuda a la osteoconducción y posterior crecimiento óseo. No es reabsorbible y, por lo tanto, no es reemplazado por hueso. El ionómero de vidrio se ha considerado como un sustitutivo del polimetilmetacrilato (PMMA) pero sin una reacción exotérmica tan significativa(3).

\section{Factores de crecimiento}

\section{Matriz ósea desmineralizada}

La DBM es hueso cortical que se ha tratado con ácido para eliminar sus componentes minerales dejando la matriz de colágeno, que reproduce la arquitectura tridimensional del hueso, facilitando y guiando la invasión, el crecimiento y la diferenciación de la célula huésped, pero no tiene capacidad de soportar carga, por lo que solo puede usarse para rellenar defectos ${ }^{(2,3,5,14)}$. La DBM mantiene los factores de crecimiento inducidos por el hueso, tales como las BMP, el factor de crecimiento insulínico (IGF), el factor de crecimiento transformante (TGF), el factor de crecimiento fibroblástico (FGF)... Poseen capacidad osteoinductora, induciendo actividad osteocondrogénica de células pluripotenciales o de fibroblastos. Al igual que hay poca evidencia de estudios nivel 1-2 que apoyen el uso de DBM como sustitutivo óseo por sí solo, también hay numerosos estudios que sugieren que la DBM enriquecida con médula ósea podría ser comparable al autoinjerto para tratar fracturas de huesos 
largos y pseudoartrosis ${ }^{(9)}$. Su uso en una mezcla de DBM-SC-vancomicina fue revisada de forma prospectiva en fracturas de calcáneo ${ }^{(18)}$ obteniendo mejores tasas de unión, estadísticamente significativas, en relación con el grupo control (sin injerto).

\section{Plasma rico en plaquetas}

Las plaquetas son una fuente de factores de crecimiento, como el factor de crecimiento derivado de plaquetas (PDGF), IGF o TGF. Ha demostrado ejercer propiedades quimiotácticas y mitogénicas para osteoblastos y fibroblastos, pero no ha sido validado como sustitutivo óseo por sí solo, siendo más bien un cofactor de crecimiento para la consolidación ósea ${ }^{(2)}$. El PDGF es un estimulador de la formación ósea que ha demostrado ser eficaz con evidencia clínica de nivel I en estudios con PDGF recombinante mezclado con $\beta$-TCP, habiendo sido aprobado para su uso clínico en artrodesis de tobillo y retropié en los Estados Unidos en 2015(19).

\section{Proteínas morfogenéticas}

Representan una amplia y heterogénea familia de proteínas secretadas, dentro de la superfamilia del TGF- $\beta$, profundamente implicadas en el proceso de formación ósea. El potencial osteogénico y osteoinductivo de las BMP ha sido ampliamente validado, con tasas de fusión similares al hueso autólogo. Solo se ha aprobado el uso de BMP-2 para fusión lumbar y fracturas abiertas de tibia, y BMP-7 para pseudoartrosis de tibia ${ }^{(2,0,16)}$. Se consideran las moléculas más potentes en la inducción de la consolidación ósea, pero a pesar de la evidencia de las BMP, no dominan las estrategias para la regeneración ósea debido a diferentes cuestiones como el limitado conocimiento de sus mecanismos funcionales, la poca claridad en relación con la dosis ideal, los prerrequisitos necesarios para su éxito (disponibilidad de las células osteoprogenitoras en la fractura o pseudoartrosis o la necesidad de un adecuado aporte sanguíneo)(10) o los altos costes.

\section{Metal trabecular}

El colapso puede ser un problema de los auto- y homoinjertos, por lo que los suplementos metá- licos se han utilizado en su lugar en cirugía reconstructiva articular o de fusión cervical. El tantalio es un metal inerte con alta resistencia a la corrosión, que se produce con indicaciones en cirugía ortopédica con una porosidad del $80 \%$ y un tamaño de los poros de 400-600 $\mu \mathrm{m}^{(20)}$. Tiene una alta biocompatibilidad y bioactividad que permite el crecimiento óseo través de él, con una gran capacidad de soporte estructural que se mantiene a lo largo del tiempo. El uso de tantalio podría ser una alternativa en las artrodesis del pie y el tobillo ${ }^{(20,21)}$.

\section{Nuevas estrategias}

\section{Materiales biomiméticos e inteligentes en la ingeniería de tejidos óseos}

El reto para los ingenieros tisulares es diseñar y desarrollar andamiajes óseos temporales que entreguen moléculas bioactivas y fármacos o células al sitio de lesión y, por lo tanto, amplíen su funcionalidad biológica(2).

La copolimerización es un proceso químico utilizado para ganar más control sobre la tasa de degradación, hidrofobicidad y cristalinidad, siendo el copolímero más utilizado para la encapsulación y liberación de moléculas bioactivas el copolímero poli (ácido láctico-ácido co-glicólico) que, aunque ha demostrado ser prometedor como armazón óseo, presenta una utilidad clínica limitada debido a sus precarias propiedades mecánicas(2).

Otros problemas de la ingeniería tisular en los sustitutivos óseos son la ausencia de eficacia para los grandes defectos óseos debida a la muerte de la mayoría de las células poco después del trasplante (las células requieren un activo aporte sanguíneo, que muchas veces está limitado), alto coste, condiciones especiales de almacenamiento no siempre disponibles en las instituciones médicas, complejidad de la legalidad al respecto de productos médicos que contienen células vivas ${ }^{(22)}$, etc.

\section{Terapia génica celular}

Los enfoques celulares se basan fundamentalmente en las células madre mesenquimales (MSC, mesenchymal stem cells), que residen en el 
estroma de la médula ósea y de otros organismos y tejidos (músculo, piel, grasa...). La gran ventaja de las MSC es su facilidad de aislamiento y obtención. Existen 3 posibles estrategias con MSC(11):

- Aspiración, centrifugado para concentrar células mononucleares y posterior implantación con o sin sustitutivo óseo sintético.

- Cultivo de las MSC obtenidas durante 2-3 semanas en una unidad de terapia celular para ampliar la fracción de MSC y posterior introducción solas o en un andamiaje.

- Aislamiento de MSC de médula ósea, expansión de los osteoprogenitores durante varias semanas y posterior colocación en un andamiaje, donde se cultivan durante unas semanas más para promover la formación de una capa de tejido óseo en el implante.

Debido a la facilidad de obtención, concentración e introducción, el aspirado de médula ósea es la técnica de terapia celular más utilizada en la regeneración ósea en cirugía ortopédica, habiéndose comprobado su utilidad en pseudoartrosis, fusión espinal y osteonecrosis ${ }^{(11)}$. Los desarrollos futuros se centrarán en mejoras en la composición del material y en los vehículos para el suministro de BMP, y es probable que impliquen un mayor uso de células pluripotenciales. El injerto óseo ideal tendrá propiedades osteoconductivas, osteoinductivas y osteogénicas similares a las del hueso autógeno(6).

Se requieren resultados clínicos completos para avanzar en las estrategias de terapia celular para la regeneración ósea y los ensayos clínicos ya iniciados pueden proporcionar información importante en un futuro próximo. Las propiedades inmunosupresoras de las MSC podrían permitir el trasplante de MSC alogénicas, con el establecimiento de bancos de células para la medicina regenerativa. Los primeros ensayos que evalúan MSC homólogas en retrasos de consolidación ya están en marcha ${ }^{(11)}$.

\section{Bioimpresión}

La capacidad de biofabricar órganos funcionales sólidos todavía no es una realidad, pero un enfoque alternativo puede ser bioimprimir una plantilla rudimentaria para la posterior organogénesis in vivo, como la conseguida por Daly et al.(23) en ratones. Este estudio muestra el desarrollo de una plantilla vertebral de cartílago hipertrófico reforzado con fibras de policaprolactona, que posteriormente se implanta subcutáneamente, apreciándose a las 12 semanas una vascularización del injerto y la formación de un tejido similar al hueso trabecular. También se han fabricado con éxito andamiajes de HA y ácido poliláctico con buena biocompatibilidad, capaces de estimular in vivo la proliferación y diferenciación de células osteogénicas del huésped ${ }^{(24)}$.

\section{Evidencias actuales de los sustitutivos óseos}

Existe un creciente interés en los sustitutivos óseos, traducido en una presencia cada vez mayor de artículos al respecto en la literatura pero, ¿qué hay de evidencia en todo este tema?

Kurian et al. ${ }^{(14)}$ intentan realizar una revisión sistemática del uso de sustitutivos óseos revisando 59 tipos diferentes pero, tras limitar la literatura a estudios clínicos, solo 22 sustitutivos pudieron ser valorados, de los que solo 4 poseen estudios con evidencia de nivel I: Norian ${ }^{\circledR}$, Vitoss ${ }^{\circledR}$, Cortoss ${ }^{\circledR}$ y Alpha-BSM ${ }^{\circledR}$. Defienden que a pesar de existir muchos estudios en animales, existen pocos datos para justificar el uso de sustitutivos óseos.

En una reciente revisión sistemática de sustitutivos óseos en osteotomías de apertura de tibia se comprueba que la consolidación ósea se obtiene antes con el uso de autoinjertos que con homoinjertos, que a su vez tienen mejores tasas de unión que los compuestos sintéticos ${ }^{(25)}$. Y en otra realizada en fusión espinal(26) se apoya que, aunque muchos sustitutivos óseos funcionan bien como osteoconductores, solo la BMP-2 y la BMP7 proporcionan evidencia de uso para utilizarlos como osteoinductores. Los hidrogeles de ingeniería tisular, los compuestos poliméricos sintéticos y la terapia génica vírica también tendrían potencial de uso en fusión vertebral, pero se necesita una mayor investigación para poder utilizarlos en la práctica clínica ${ }^{(26)}$. En otra revisión de fracturas osteoporóticas, Lieshout y Alt ${ }^{(16)}$ concluyen que es decepcionante observar que existen muy pocos datos en relación con los sustitutivos óseos y BMP, que no permiten realizar un algoritmo basado en la evidencia en esta patología, por lo que animan a los investigadores a centrarse en estos materiales y su uso en fracturas osteoporóticas. 
Roffi et al.(27) realizan una amplia revisión de la literatura relativa al PRP. Aprecian un alto número de estudios de baja calidad en la literatura, pero también un creciente interés en los sustitutivos óseos. De todos los artículos revisados, 16 apoyaban el uso de PRP, 18 no obtuvieron diferencias y 6 cuestionaban la utilidad del mismo, por lo que concluyen que no existe clara evidencia para su uso como sustitutivo.

Handoll y Watts(28) realizan una revisión sistemática sobre sustitutivos óseos en fracturas de radio apreciando evidencias sobre los mejores resultados con autoinjerto, pero no existen suficientes evidencias que apoyen el uso de sustitutivos óseos. No obstante, admiten que los mejores resultados obtenidos con Norian ${ }^{\circledR}$ pueden ser ciertos, si bien es un estudio patrocinado por la empresa que lo comercializa.

En otra revisión Cochrane ${ }^{(29)}$ en fracturas de meseta tibial se concluye que no hay pruebas suficientes para determinar el mejor método de tratamiento de los defectos óseos, pero las pruebas existentes no contradicen los enfoques que intenten limitar el daño del sitio donante.

\section{Evidencias en pie y tobillo de los sustitutivos óseos}

Müller et al. ${ }^{(30)}$ realizan una revisión sistemática de sustitutivos óseos en artrodesis y osteotomías del retropié, haciendo hincapié en la necesidad de elegir el tipo de sustitutivo óseo basándose no solo en las propiedades biológicas y biomecánicas, sino también en la evidencia existente en la literatura. Indican que la evidencia actual sugiere que los homoinjertos estructurales parecen ser similares a los autoinjertos en tasas de fusión y que la DBM y los gránulos de cerámica de $\beta$-TCP suplementado con factores de crecimiento plaquetarios (PDGF) podrían ser buenos sustitutivos óseos para las fusiones del retropié, apuntando, no obstante, la ausencia de estudios prospectivos con buena metodología que apoyen estas ideas.

Krelly y DiGiovanni ${ }^{(19)}$ realizan una revisión de PDGF recombinante mezclado con $\beta$-TCP sugiriendo que el homoinjerto autólogo podría no ser más el injerto óptimo para cirugía compleja ortopédica y que el PDGF recombinante mezclado con $\beta$-TCP (Augment ${ }^{\circledR}$ ) presenta un nivel de evidencia I como alternativa para facilitar la fusión en ar- trodesis de retropié y tobillo, a la vez de evitar el dolor y la morbilidad de la zona donante.

Arner y Santrock ${ }^{(8)}$ realizan una reciente revisión de sustitutivos óseos concluyendo que, aunque se ha demostrado que tanto el DBM como los PDGF son seguros y tienen tasas de fusión aceptables, hacen falta muchos estudios para poder recomendar su uso generalizado. Aunque hallan resultados prometedores en 2 estudios realizados con HA coralina y con DBM, no encuentran datos que apoyen el uso de sustitutivos óseos en comparación con el injerto autólogo de cresta iliaca y apuntan que existe abundante literatura de baja calidad y patrocinada por la industria farmacéutica. El importante coste de estos productos añadido a la ausencia de evidencia clínica de los estudios de pie y tobillo les impide realizar ninguna recomendación aparte del autoinjerto(8).

Glazebrook y Young ${ }^{(31)}$ apoyan el uso del B2A, un péptido sintético bioactivo que aumenta la osteodiferenciación mediante el aumento local de BMP-2 endógena. El gránulo B2A es comparable con el injerto óseo autólogo con respecto a la seguridad y eficacia, con similares tasas de fusión en artrodesis de pie y tobillo, eliminando el riesgo de morbilidad en el sitio del donante. Aunque el estudio realizado es multicéntrico, prospectivo y aleatorizado ${ }^{(32)}$, el gránulo B2A aún no se ha probado en ensayos a gran escala, que ha sido aprobado para iniciarse en 2017.

Los PDGF recombinantes tienen un enorme potencial angiogénico, mitogénico y quimiotáctico, por lo que son osteoinductivos. Su combinación con una superficie osteoconductora como el fosfato tricálcico ha sido recientemente valorada por Digiovani et al. ${ }^{(33)}$ en un estudio prospectivo aleatorizado y multicéntrico, con nivel de evidencia I, en pacientes sometidos a artrodesis de tobillo o retropié, obteniendo tasas de unión, tiempo hasta la unión y resultados clínicos comparables al autoinjerto. Asimismo, el grupo con TCP demostró una reducción del tiempo quirúrgico del $22 \%$ en comparación con el grupo de autoinjerto óseo, además de evitar la morbilidad y el dolor del sitio donante.

\section{Conclusiones}

Existen resultados prometedores con el uso de algunos sustitutivos óseos como las BMP, la DBM 
y los PDGF combinados con $\beta$-TCP. No obstante, el autoinjerto sigue siendo hoy en día el patrón oro debido a que posee capacidad osteogénica, osteoinductiva y osteoconductiva, aunque este hecho podría dejar de ser así en un futuro, debido a la aparición de nuevas evidencias que apoyen el uso de otros sustitutivos que eviten la morbilidad de la zona donante con iguales o mejores resultados clínicos.

Hay poca evidencia en relación con el uso generalizado de sustitutivos óseos, con una gran ausencia de estudios bien diseñados, sobre todo en la patología de pie y tobillo, siendo mucho más frecuente este estudio en la odontología o en fusión espinal. Sería conveniente la realización de estudios bien diseñados y no patrocinados por la industria farmacéutica para apoyar o no el uso de sustitutivos óseos.

\section{Bibliografía}

1. Van Lieshout EMM, Van Kralingen GH, El-Massoudi Y, Weinans H, Patka P. Microstructure and biomechanical characteristics of bone substitutes for trauma and orthopaedic surgery. BMC Musculoskelet Disord. 2011;12:34.

2. Campana V, Milano G, Pagano E, Barba M, Cicione C, Salonna $G$, et al. Bone substitutes in orthopaedic surgery: from basic science to clinical practice. J Mater Sci Mater Med. 2014;25(10):2445-61.

3. Giannoudis P V, Dinopoulos H, Tsiridis E. Bone substitutes: an update. Injury. 2005;36 Suppl 3:S20-7.

4. Chiarello E, Cadossi M, Tedesco G, Capra P, Calamelli C, Shehu A, et al. Autograft, allograft and bone substitutes in reconstructive orthopedic surgery. Aging Clin Exp Res. 2013;25 Suppl 1:S101-3.

5. Cook EA, Cook JJ. Bone graft substitutes and allografts for reconstruction of the foot and ankle. Clin Podiatr Med Surg. 2009;26(4):589-605.

6. Fillingham $Y$, Jacobs J. Bone grafts and their substitutes. Bone Joint J. 2016;98-B(1 Suppl A):6-9.

7. Hannink G, Arts JJC. Bioresorbability, porosity and mechanical strength of bone substitutes: what is optimal for bone regeneration? Injury. 2011;42 Suppl 2:S22-5.

8. Arner JW, Santrock RD. A historical review of common bone graft materials in foot and ankle surgery. Foot Ankle Spec. 2014;7(2):143-51.

9. Egol KA, Nauth A, Lee M, Pape H-C, Watson JT, Borrelli J. Bone Grafting: Sourcing, Timing, Strategies, and Alternatives. J Orthop Trauma. 2015;29 Suppl 1:S10-4.
10. Nauth A, Lane J, Watson JT, Giannoudis P. Bone Graft Substitution and Augmentation. J Orthop Trauma. 2015;29 Suppl 1:S34-8.

11. Stanovici J, Le Nail L-R, Brennan MA, Vidal L, Trichet V, Rosset $\mathrm{P}$, et al. Bone regeneration strategies with bone marrow stromal cells in orthopaedic surgery. Curr Res Transl Med. 2016;64(2):83-90.

12. Coughlin MJ, Grimes IS, Kennedy MP. Coralline hydroxyapatite bone graft substitute in hindfoot surgery. Foot ankle Int. 2006;27(1):19-22.

13. Huber FX, Hillmeier J, McArthur N, Kock HJ, Meeder PJ. The use of nanocrystalline hydroxyapatite for the reconstruction of calcaneal fractures: Preliminary results. J Foot Ankle Surg. 2006;45(5):322-8.

14. Kurien T, Pearson RG, Scammell BE. Bone graft substitutes currently available in orthopaedic practice: The evidence for their use. Bone Joint J. 2013;95-B(5): 583-97.

15. O'Neill R, McCarthy HO, Montufar E, Ginebra M-P, Wilson DI, Lennon A, et al. Critical Review: Injectability of Calcium Phosphate Pastes and Cements. Acta Biomater. 2017;50:1-19.

16. Van Lieshout EMM, Alt V. Bone graft substitutes and bone morphogenetic proteins for osteoporotic fractures: what is the evidence? Injury. 2016;47 Suppl 1: S43-6.

17. Dewi AH, Ana ID, Wolke J, Jansen J. Behavior of plaster of Paris-calcium carbonate composite as bone substitute. A study in rats. J Biomed Mater Res A. 2013;101(8):2143-50.

18. Bibbo C, Patel D V. The effect of demineralized bone matrix-calcium sulfate with vancomycin on calcaneal fracture healing and infection rates: a prospective study. Foot ankle Int. 2006;27(7):487-93.

19. Krell ES, DiGiovanni CW. The Efficacy of Platelet-Derived Growth Factor as a Bone-Stimulating Agent. Foot Ankle Clin. 2016;21(4):763-70.

20. Sagherian BH, Claridge RJ. Porous tantalum as a structural graft in foot and ankle surgery. Foot ankle Int. 2012;33(3):179-89.

21. Frigg A, Dougall H, Boyd S, Nigg B. Can porous tantalum be used to achieve ankle and subtalar arthrodesis?: a pilot study. Clin Orthop Relat Res. 2010;468(1):209-16.

22. Deev R V, Drobyshev AY, Bozo IY, Isaev AA. Ordinary and Activated Bone Grafts: Applied Classification and the Main Features. Biomed Res Int. 2015;2015:365050.

23. Daly AC, Cunniffe GM, Sathy BN, Jeon O, Alsberg E, Kelly DJ. 3D Bioprinting of Developmentally Inspired Templates for Whole Bone Organ Engineering. Adv Healthc Mater. 2016;5(18):2353-62. 
24. Zhang H, Mao X, Du Z, Jiang W, Han X, Zhao D, et al. Three dimensional printed macroporous polylactic acid/hydroxyapatite composite scaffolds for promoting bone formation in a critical-size rat calvarial defect model. Sci Technol Adv Mater. 2016;17(1):136-48.

25. Lash NJ, Feller JA, Batty LM, Wasiak J, Richmond AK. Bone grafts and bone substitutes for opening-wedge osteotomies of the knee: a systematic review. Arthroscopy. 2015;31(4):720-30.

26. Gupta A, Kukkar N, Sharif K, Main BJ, Albers CE, El-Amin lii SF. Bone graft substitutes for spine fusion: A brief review. World J Orthop. 2015;6(6):449-56.

27. Roffi A, Filardo G, Kon E, Marcacci M. Does PRP enhance bone integration with grafts, graft substitutes, or implants? A systematic review. BMC Musculoskelet Disord. 2013;14:330.

28. Handoll HH, Watts AC. Bone grafts and bone substitutes for treating distal radial fractures in adults. Cochrane Database Syst Rev. 2008;(2).
29. Métodos de fijación quirúrgica para la fractura de la meseta tibial (Revision Cochrane traducida) Cochrane Database Syst Rev. 2015 Sep 1;(9).

30. Müller MA, Frank A, Briel M, Valderrabano V, Vavken $P$, Entezari $V$, et al. Substitutes of structural and non-structural autologous bone grafts in hindfoot arthrodeses and osteotomies: a systematic review. BMC Musculoskelet Disord. 2013;14:59.

31. Glazebrook M, Young DS. B2A Polypeptide in Foot and Ankle Fusion. Foot Ankle Clin. 2016;21(4):803-7.

32. Glazebrook M, Younger A, Wing K, Lalonde KA. A prospective pilot study of B2A-coated ceramic granules (Amplex) compared to autograft for ankle and hindfoot arthrodesis. Foot ankle Int. 2013;34(8):1055-63.

33. Digiovanni CW, Baumhauer J, Lin SS, Berberian WS, Flemister AS, Enna MJ, et al. Prospective, randomized, multi-center feasibility trial of rhPDGF-BB versus autologous bone graft in a foot and ankle fusion model. Foot Ankle Int. 2011;32(4):344-54. 